\title{
Salivary Duct Inflammation, CTCAE
}

National Cancer Institute

\section{Source}

National Cancer Institute. Salivary Duct Inflammation, CTCAE. NCI Thesaurus. Code C143821.

A disorder characterized by inflammation of the salivary duct. 